\title{
Renal Outcomes in Brazilian Patients with Immunoglobulin A Nephropathy and Cellular Crescentic Lesions
}

\author{
Precil Diego Miranda de Menezes Neves ${ }^{a} \quad$ Rafaela Bezerra Brito Pinheiro ${ }^{b}$ \\ Cristiane Bitencourt Dias ${ }^{a}$ Luis $\mathrm{Yu}^{a}$ Leonardo de Abreu Testagrossa ${ }^{b}$ \\ Lívia Barreira Cavalcante $^{b}$ Denise Maria Avancini Costa Malheiros ${ }^{b}$ \\ Lectícia Barbosa Jorge $^{a}$ Viktoria Woronik ${ }^{a}$

\footnotetext{
a Nephrology Department, University of São Paulo School of Medicine, São Paulo, Brazil;

bPathology Department, University of São Paulo School of Medicine, São Paulo, Brazil
}

\section{Keywords}

Immunoglobulin A nephropathy · Kidney biopsy · Pathology · End-stage renal disease

\begin{abstract}
Background and Aim: Immunoglobulin A nephropathy (IgAN) is the most common primary glomerulopathy. The Oxford classification was recently updated to include crescents as markers of poor prognosis. The aim of this study was to evaluate the impact of cellular crescents on the prognosis of patients with IgAN in Brazil. Methods: This was a single-centre retrospective analysis of medical records and renal biopsies in patients with IgAN. The renal biopsy findings were classified according to the revised Oxford classification: mesangial hypercellularity, endocapillary hypercellularity $(E)$, segmental glomerulosclerosis $(S)$, tubular atrophy or interstitial fibrosis $(\mathrm{T})$, and crescent formation (C). We evaluated a composite outcome (progression to end-stage renal disease or creatinine doubling). We performed analyses between the patients with crescents in the renal biopsy specimen (C1/C2 group) and those without such crescents (CO group). Results: We evaluated 111 patients, of whom 72 (65.0\%) were women, 80 (72.0\%) self-identified as White, 73 (65.6\%) were hypertensive, and 95 (85.6\%) had haematuria. The distribution of patients according to cellular crescentic lesions was: $\mathrm{C0}, 80$ (72\%); C1, 27 (24.4\%); C2, 4 (3.6\%). The composite outcome was observed in 33 (29.72\%) of the 111 patients. In comparison with the $\mathrm{C} 0$ group, the $\mathrm{C} 1 / \mathrm{C} 2$ group had higher proportions of patients with hypertension $(p=0.04)$, haematuria $(p=0.03)$, worse serum creatinine $(p=0.0007)$, and worse estimated glomerular filtration rate $(p=0.0007)$. The $C 1 / C 2$ group also had higher proportions of patients in whom the biopsy specimen was classified as E1 $(p=0.009), \mathrm{S} 1$ ( $p=$
\end{abstract}




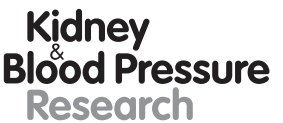

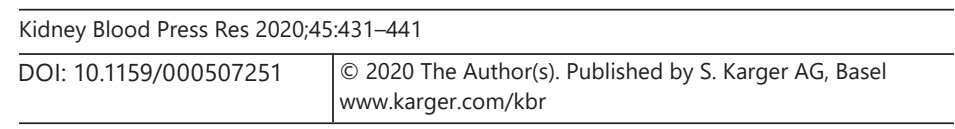

Neves et al.: Immunoglobulin A Nephropathy and Cellular Crescents

$0.001)$, or $\mathrm{T} 1 / \mathrm{T} 2$ ( $p=0.03$ ), In addition, the mean follow-up period was shorter in the C1/C2 group ( $p<0.0001)$. Furthermore, the composite outcome was observed in a greater proportion of patients and in a shorter length of time in the $\mathrm{C} 1 / \mathrm{C} 2$ group than in the $\mathrm{C} 0$ group $(p=$ 0.002 and $p=0.0014$, respectively). In a Cox regression analysis, the independent risk factors for the composite outcome had Oxford classifications of $\mathrm{S} 1, \mathrm{~T} 1 / \mathrm{T} 2$, and $\mathrm{C} 1 / \mathrm{C} 2$. Conclusion: Oxford classification findings of $\mathrm{S} 1, \mathrm{~T} 1 / \mathrm{T} 2$, or $\mathrm{C} 1 / \mathrm{C} 2$ were independent risk factors for the composite outcome, corroborating previous studies.

(C) 2020 The Author(s)

Published by S. Karger AG, Basel

\section{Introduction}

Immunoglobulin A nephropathy (IgAN) is the most common primary glomerulopathy [1-3]. Its prevalence varies according to geographic region, being higher in the Asian and Pacific regions; it is also more prevalent among White individuals, whereas it is uncommon among Black individuals $[4,5]$. The clinical presentations of IgAN include asymptomatic microscopic haematuria, pure nephrotic syndrome, and rapid loss of function in the form of rapidly progressive glomerulonephritis $[1,6,7]$.

Approximately $30 \%$ of IgAN cases evolve to end-stage renal disease (ESRD) within 5 years $[1,4]$. Given the prevalence of the disease, that leads to a need of identifying risk factors related to a poorer prognosis. A worse prognosis in IgAN has been associated with male sex, smoking, obesity, hypertension, renal failure, and severe proteinuria $[1,4,8]$.

In 2009, the Working Group of the International IgA Nephropathy Network and the Renal Pathology Society published the Oxford classification of IgAN histology $[9,10]$. The Oxford classification is aimed at identifying the histological markers related to a poorer prognosis. Among the markers identified were mesangial hypercellularity, segmental glomerulosclerosis, and tubular atrophy or interstitial fibrosis.

After the publication of the original Oxford classification, a number of studies highlighted the importance of cellular/fibrocellular crescents (hereafter crescents) as contributors to a poorer prognosis in IgAN [11-13]. Consequently, the IgA Nephropathy Classification Working Group published an updated version of the Oxford classification in 2017 [14]. In the updated version, crescents are recognised as markers of poor prognosis in IgAN. The objective of the present study was to evaluate the impact that a finding of crescents has on the prognosis of patients with IgAN in Brazil.

\section{Subjects and Methods}

\section{Study Design and Study Population}

This was a retrospective single-centre study of patients diagnosed with IgAN based on renal biopsy findings. The biopsies were performed and analysed in the Glomerulopathy Division of the Nephrology Department and in the Pathology Department of University of São Paulo School of Medicine in the city of São Paulo, Brazil between January 1996 and December 2016. Patients with IgAN secondary to systemic diseases (e.g., liver disease, HIV infection, and Henoch-Schönlein purpura) were excluded, as were those for whom there was insufficient clinical follow-up information and those for whom there were $<8$ glomeruli in the renal biopsy specimen.

At biopsy, the following demographic and clinical data were evaluated: sex, age, race, serum creatinine, glomerular filtration rate, 24 -h proteinuria or the protein/creatinine ratio in an isolated sample, haematuria, hypertension, serum IgA, serum C3, and the IgA/C3 ratio. 


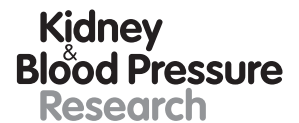

Kidney
Blood Pressure
Research

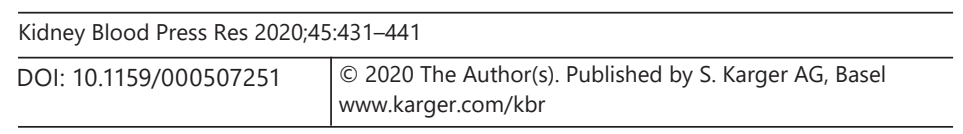

Neves et al.: Immunoglobulin A Nephropathy and Cellular Crescents

Because of the extensive miscegenation in Brazil, self-reported skin colour was used as a proxy for race, the patients thus being categorised as White, non-White, or Yellow. At hospital admission for renal biopsy, haematuria was defined as $\geq 5$ red blood cells/field in the urine sample and hypertension was defined as systolic blood pressure $\geq 140 \mathrm{~mm} \mathrm{Hg}$ and/or diastolic blood pressure $\geq 90 \mathrm{~mm} \mathrm{Hg}$. The estimated glomerular filtration rate (eGFR) was determined by the Chronic Kidney Disease Epidemiology Collaboration (CKD-EPI) equation as previously described [15]. The serum levels of C3, IgA, and haptoglobin were evaluated by immunoturbidimetric assay, the normal ranges being 90-180, 69-382, and 30-200 mg/dL, respectively. Lactate dehydrogenase activity was analysed by kinetic assay, in accordance with the International Federation of Clinical Chemistry guidelines, with a reference range of 135-214 U/L.

Every 3-6 months, the clinical treatment (use of angiotensin-converting enzyme inhibitors or angiotensin II receptor blockers and use of corticosteroids or other immunosuppressants) was evaluated, as was the evolution of clinical parameters (glomerular filtration rate, proteinuria, and haematuria). The end of follow-up was defined as the last visit to the clinic, referral for dialysis, or kidney transplantation.

\section{Histopathological Evaluation}

All renal biopsies were reviewed by a nephropathologist and reclassified in accordance with the 2017 Oxford classification [14], which considers cellular crescents as histological prognostic markers. As previously mentioned, the presence of at least 8 glomeruli in the renal biopsy specimen was sine qua non for inclusion in the sample. To best characterise cellular crescents, we divided lesions according to the extent of glomerular affection (segmental vs. global) and to the number of glomeruli involved in tissue sample (focal vs. diffuse).

\section{Analysis of the Outcomes}

The primary outcomes evaluated were progression to ESRD and doubling of the baseline serum creatinine concentration, the two being evaluated together as a composite outcome. The secondary outcomes were reaching CKD-EPI-determined eGFR $\leq 60 \mathrm{~mL} / \mathrm{min} / 1.73 \mathrm{~m}^{2}$.

\section{Statistical Analysis}

To evaluate the distribution of data, the D'Agostino-Pearson omnibus test was applied. Variables with normal data distribution were expressed as mean \pm standard deviation and were compared by using the Student $t$ test. Variables with non-normal data distribution were expressed as median (interquartile range) and were compared by using the Mann-Whitney test. Qualitative variables were expressed as percentages and analysed by $\chi^{2}$ or Fisher exact test. Multivariate analysis was performed by Cox regression analysis. $p$ values $<0.05$ were considered statistically significant. Statistical analyses were performed using GraphPad Prism, version 7.00 for Windows (GraphPad Software, La Jolla, CA, USA; www.graphpad.com) or the SPSS Statistics software package, version 25.0 (IBM Corp., Armonk, NY, USA).

\section{Results}

From an initial sample of 154 patients, 43 were excluded. Therefore, the final sample comprised 111 patients with at least 8 glomeruli in the renal biopsy specimen. There was a predominance of women and Whites, who accounted for 65 and $72 \%$ of the sample, respectively. In addition, $65.6 \%$ of the patients were hypertensive and $85.6 \%$ presented with haematuria. The histological findings (Oxford classifications) were as follows: mesangial hypercellularity (M1), 77.5\%; endocapillary hypercellularity (E1), 38.7\%; segmental glomerulosclerosis (S1), 76.6\%; tubular atrophy or interstitial fibrosis (T1/T2), 41.4\%; and crescent formation 
Table 1. Demographic and clinical characteristics, together with renal biopsy findings, in patients with IgAN $(n=111)$

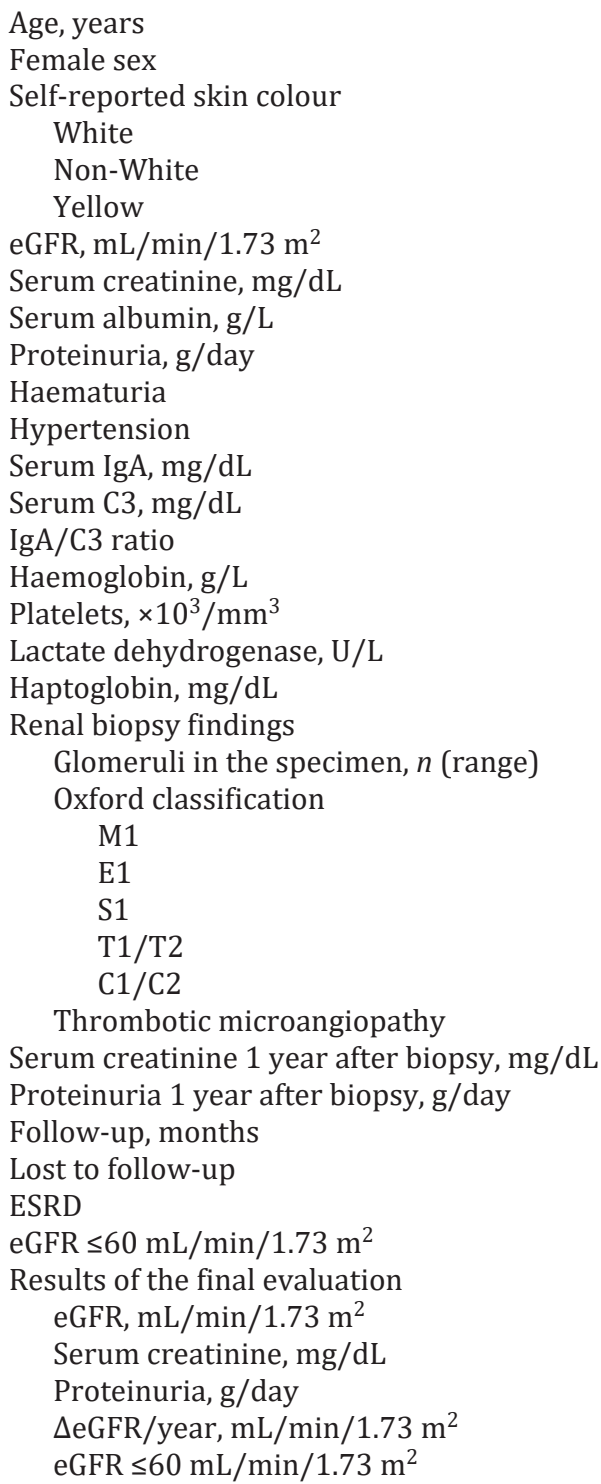

Thrombotic microangiopathy

Serum creatinine 1 year after biopsy, mg/dL

Proteinuria 1 year after biopsy, g/day

Follow-up, months

Lost to follow-up

ESRD

eGFR $\leq 60 \mathrm{~mL} / \mathrm{min} / 1.73 \mathrm{~m}^{2}$

Results of the final evaluation

eGFR, $\mathrm{mL} / \mathrm{min} / 1.73 \mathrm{~m}^{2}$

Serum creatinine, $\mathrm{mg} / \mathrm{dL}$

Proteinuria, g/day

$\Delta$ eGFR/year, $\mathrm{mL} / \mathrm{min} / 1.73 \mathrm{~m}^{2}$

eGFR $\leq 60 \mathrm{~mL} / \mathrm{min} / 1.73 \mathrm{~m}^{2}$

Values are expressed as median (interquartile range), $n(\%)$, or mean \pm standard deviation unless indicated otherwise. eGFR was determined by the Chronic Kidney Disease Epidemiology Collaboration equation. C1/C2, pronounced/severe crescent formation; E1, pronounced endocapillary hypercellularity; eGFR, estimated glomerular filtration rate; ESRD, end-stage renal disease; IgA, immunoglobulin A; IgAN, immunoglobulin A nephropathy; M1, pronounced mesangial hypercellularity; S1, pronounced segmental glomerulosclerosis; T1/T2, pronounced/severe tubular atrophy or interstitial fibrosis.

(C1/C2), 28.0\%. The distribution of patients according to cellular crescentic lesions was: C0, 80 (72\%); C1, 27 (24.4\%); and C2, 4 (3.6\%). Analyses of 27 from 31 patients allowed characterisation of cellular crescent distribution in kidney biopsy: 88.8\% focal and segmental, 7.4\% focal and global, and 3.8\% global and diffuse. The median follow-up period was 64 months, during which time 31 patients (29.7\%) evolved to ESRD. Other clinical findings, demographic characteristics, and laboratory test results at the time of renal biopsy are shown in Table 1. 
Table 2. Characteristics of patients with IgAN, with and without crescents

\begin{tabular}{lccc}
\hline & C0 $(n=80)$ & C1/C2 $(n=31)$ & $p$ value \\
\hline Age, years & $33(25-47)$ & $32(24-42)$ & 0.54 \\
Male sex & $37(46.0 \%)$ & $18(58.0 \%)$ & 0.29 \\
Hypertension & $31(39.3 \%)$ & $19(61.3 \%)$ & 0.04 \\
Haematuria & $65(81.0 \%)$ & $30(96.7 \%)$ & 0.03 \\
Serum creatinine, mg/dL & $1.27(0.9-2.0)$ & $1.89(1.38-2.9)$ & 0.0007 \\
eGFR, mL/min/1.73 m ${ }^{2}$ & $65(33.5-88.7)$ & $36(23-54.89)$ & 0.0007 \\
Proteinuria, g/day & $1.5(1.1-3.1)$ & $2.38(1.4-4.1)$ & 0.09 \\
Serum albumin, g/L & $3.5(3-3.9)$ & $3.3(3.2-3.6)$ & 0.2 \\
High serum IgA & $20(25.0 \%)$ & $15(47.8 \%)$ & 0.02 \\
Low serum C3 & $13(16.8 \%)$ & $4(13.7 \%)$ & 1 \\
IgA/C3 ratio & $2.9(2.3-4.6)$ & $2.8(2.3-3.3)$ & 0.65 \\
Oxford classification & & & 0.44 \\
$\quad$ M1 & $60(75.0 \%)$ & $26(83.0 \%)$ & 0.009 \\
$\quad$ E1 & $23(28.7 \%)$ & $20(64.5 \%)$ & 0.001 \\
$\quad$ S1 T1/T2 & $55(68.7 \%)$ & $30(96.7 \%)$ & 0.03 \\
Follow-up, months & $28(35.0 \%)$ & $18(58.0 \%)$ & $<0.0001$ \\
ESRD or creatinine doubling & $70(35.5-121.5)$ & $11(4-65.7)$ & 0.002 \\
eGFR $\leq 60$ mL/min/1.73 m ${ }^{2}$ & $17(21.2 \%)$ & $16(51.6 \%)$ & 0.001 \\
Time to ESRD, months & $38(47.5 \%)$ & $25(80.6 \%)$ & 0.0014 \\
\hline
\end{tabular}

Values are expressed as median (interquartile range) or $n(\%)$ unless indicated otherwise. eGFR was determined by the Chronic Kidney Disease Epidemiology Collaboration equation. C0, no crescent formation; C1/C2, pronounced/severe crescent formation; E1, pronounced endocapillary hypercellularity; eGFR, estimated glomerular filtration rate; ESRD, end-stage renal disease; IgA, immunoglobulin A; IgAN, immunoglobulin A nephropathy; M1, pronounced mesangial hypercellularity; S1, pronounced segmental glomerulosclerosis; T1/T2, pronounced/severe tubular atrophy or interstitial fibrosis.

Comparing the patients with and without crescents (Oxford classifications of C1/C2 and C0, respectively) at the time of renal biopsy (Table 2), we observed that the proportion of hypertensive patients was higher in the former group (61.3 vs. 39.3\%, $p=0.04$ ), as was the proportion of patients with haematuria ( 96.7 vs. $81.0 \%, p=0.03$ ). At biopsy, the median serum creatinine concentration was also worse in the C1/C2 group (1.89 vs. $1.27 \mathrm{mg} / \mathrm{dL}, p=$ 0.0007), as was the median CKD-EPI-determined eGFR (36 vs. $65 \mathrm{~mL} / \mathrm{min} / 1.73 \mathrm{~m}^{2}, p=$ $0.0007)$, and the proportion of patients with an elevated serum IgA level was higher in the C1/C2 group ( 47.8 vs. $25.0 \%, p=0.02$ ). At renal biopsy, the C1/C2 group also had higher proportions of patients with an Oxford classifications of E1 (64.5 vs. $28.7 \%, p=0.009$ ), S1 ( 96.7 vs. $68.7 \%, p=0.001$ ), and T1/T2 (58.0 vs. $35.0 \%, p=0.03$ ). Follow-up was also shorter in the $\mathrm{C} 1 / \mathrm{C} 2$ group than in the $\mathrm{C} 0$ group (11 vs. 70 months, $p<0.0001$ ), more patients in the former group progressing to the composite outcome of ESRD or doubling of serum creatinine ( 51.6 vs. $21.2 \%, p=0.002$ ), and doing so in a shorter time ( 4.5 vs. 39.0 months, $p=0.0014$ ). Regarding the use of medications (Table 3), isolated use of an anti-proteinuric agent was more common in the C0 group (46.5 vs. $4.7 \%, p=0.0004$ ), whereas the combined use of an anti-proteinuric agent, a corticosteroid, and another immunosuppressant was more common in the $\mathrm{C} 1 / \mathrm{C} 2$ group (38.1 vs. $0 \%, p<0.0001$ ). In relation to the evolution of patients with endocapillary proliferation (E1), there was a greater proportion of patients who reached the composite outcome in the group with crescents compared to those without (39 vs. 23\%), with a tendency to statistical significance $(p=0.07)$. 


\section{Research}

Neves et al.: Immunoglobulin A Nephropathy and Cellular Crescents

Table 3. Treatment regimens employed in patients with and without crescents at renal biopsy

\begin{tabular}{lccc}
\hline Treatment & C0 $(n=58)$ & C1/C2 $(n=21)$ & $p$ value \\
\hline ACEI or ARB only & $27(46.5 \%)$ & $1(4.7 \%)$ & 0.0004 \\
ACEI or ARB + corticosteroids & $30(51.7 \%)$ & $7(33.3 \%)$ & 0.14 \\
ACEI or ARB + corticosteroids + other ISDs & $0(0.0 \%)$ & $8(38.1 \%)$ & $<0.0001$ \\
ACEI or ARB + other ISDs & $1(1.7 \%)$ & $2(9.52 \%)$ & 0.10 \\
Corticosteroids only & $0(0.0 \%)$ & $0(0.0 \%)$ & 1 \\
Corticosteroids + other ISDs & $0(0.0 \%)$ & $3(14.2 \%)$ & 0.003 \\
\hline
\end{tabular}

Values are expressed as $n$ (\%). ACEI, angiotensin-converting enzyme inhibitor; ARB, angiotensin II receptor blocker; $\mathrm{C} 0$, no crescent formation; $\mathrm{C} 1 / \mathrm{C} 2$, pronounced/severe crescent formation; ISDs, immunosuppressive drugs (azathioprine or cyclophosphamide).

Table 4. Composite outcome (ESRD or serum creatinine doubling) in patients with IgAN

\begin{tabular}{|c|c|c|c|}
\hline & \multicolumn{3}{|c|}{ ESRD or creatinine duplication } \\
\hline & no $(n=78)$ & yes $(n=33)$ & $p$ value \\
\hline Age, years & $34(26-48)$ & $29(24-37)$ & 0.038 \\
\hline Male sex & $29(37.1 \%)$ & $21(63.6 \%)$ & 0.017 \\
\hline Creatinine, mg/dL & $1.2(0.9-1.6)$ & $2.7(1.8-3.7)$ & $<0.0001$ \\
\hline $\mathrm{eGFR}, \mathrm{mL} / \mathrm{min} / 1.73 \mathrm{~m}^{2}$ & $66.8 \pm 30.9$ & $36.6 \pm 29$ & $<0.0001$ \\
\hline Proteinuria, g/day & $1.6(1.06-2.9)$ & $2.43(1.34-3.88)$ & 0.0793 \\
\hline Albumin, $\mathrm{g} / \mathrm{L}$ & $3.5(3.07-3.88)$ & $3.21(2.87-3.52)$ & 0.65 \\
\hline Haematuria & $67(85.7 \%)$ & $28(84.4 \%)$ & 1 \\
\hline Hypertension & $43(55 \%)$ & $28(84.4 \%)$ & 0.005 \\
\hline Serum creatinine 1 year after biopsy, mg/dL & $1.04(0.84-1.61)$ & $2.4(1.7-3.3)$ & $<0.0001$ \\
\hline Proteinuria 1 year after biopsy, g/day & $0.59(0.22-1.1)$ & $1.2(0.6-3.6)$ & 0.0059 \\
\hline Serum immunoglobulin $\mathrm{A}, \mathrm{mg} / \mathrm{dL}$ & $376 \pm 135$ & $403.2 \pm 177$ & 0.7957 \\
\hline Serum C3, mg/dL & $125.5 \pm 39.3$ & $110.7 \pm 21.3$ & 0.0255 \\
\hline Immunoglobulin $\mathrm{A} / \mathrm{C} 3$ ratio & $3.06(2.24-3.87)$ & $2.8(2.6-4.9)$ & 0.4921 \\
\hline Haemoglobin, g/L & $12.85 \pm 1.91$ & $12.4 \pm 2.28$ & 0.21 \\
\hline Platelets, $\times 10^{3} / \mathrm{mm}^{3}$ & $269.4 \pm 69.8$ & $251.4 \pm 100.5$ & 0.063 \\
\hline Lactate dehydrogenase, U/L & 350 (289-399) & $360(318-453)$ & 0.36 \\
\hline Haptoglobin, mg/dL & $153(94-215)$ & $133(32-166)$ & 0.1841 \\
\hline \multicolumn{4}{|l|}{ Oxford classification } \\
\hline M1 & 59 (75.3\%) & 27 (81.25\%) & 0.93 \\
\hline E1 & $26(33.8 \%)$ & $17(51.1 \%)$ & 0.11 \\
\hline S1 & $52(66.2 \%)$ & $33(100 \%)$ & 0.0002 \\
\hline $\mathrm{T} 1 / \mathrm{T} 2$ & $20(25.9 \%)$ & $26(78.1 \%)$ & $<0.0001$ \\
\hline $\mathrm{C} 1 / \mathrm{C} 2$ & $16(20.8 \%)$ & $16(46.8 \%)$ & 0.006 \\
\hline Follow-up, months & $72(42-121)$ & $16(4-51)$ & 0.0002 \\
\hline \multicolumn{4}{|l|}{ Results of the final evaluation } \\
\hline eGFR, $\mathrm{mL} / \mathrm{min} / 1.73 \mathrm{~m}^{2}$ & $68.93 \pm 33.8$ & $9.6 \pm 6.5$ & $<0.0001$ \\
\hline Creatinine, mg/dL & $1.07(0.8-1.5)$ & $7.13(5.2-10.1)$ & $<0.0001$ \\
\hline Proteinuria, g/day & $0.43(0.2-0.81)$ & $2.6(1.1-4.1)$ & $<0.0001$ \\
\hline \multicolumn{4}{|l|}{ Treatment } \\
\hline ACEI or ARB only & $64(82.5 \%)$ & $24(73 \%)$ & 0.17 \\
\hline Corticosteroids & $29(37.1 \%)$ & $19(58 \%)$ & 0.05 \\
\hline Other ISDs & $11(13.7 \%)$ & $11(33.3 \%)$ & 0.019 \\
\hline
\end{tabular}

Values are expressed as median (interquartile range), $n(\%)$, or mean \pm standard deviation unless indicated otherwise. eGFR was determined by the Chronic Kidney Disease Epidemiology Collaboration equation. ACEI, angiotensin-converting enzyme inhibitor; ARB, angiotensin II receptor blocker; C1/C2, pronounced/severe crescent formation; E1, pronounced endocapillary hypercellularity; eGFR, estimated glomerular filtration rate; ESRD, endstage renal disease; IgAN, immunoglobulin A nephropathy; ISDs, immunosuppressive drugs; M1, pronounced mesangial hypercellularity; S1, pronounced segmental glomerulosclerosis; T1/T2, pronounced/severe tubular atrophy or interstitial fibrosis. 
Fig. 1. Kaplan-Meier curve for survival free of the composite outcome (ESRD or doubling of serum creatinine) in patients with immunoglobulin A nephropathy. $\mathrm{C} 0$, no crescent formation; $\mathrm{C} 1 / \mathrm{C} 2$, crescent formation; ESRD, endstage renal disease.

Table 5. Clinical and histological risk factors for the composite outcome (ESRD or doubling of serum creatinine) in patients with IgAN

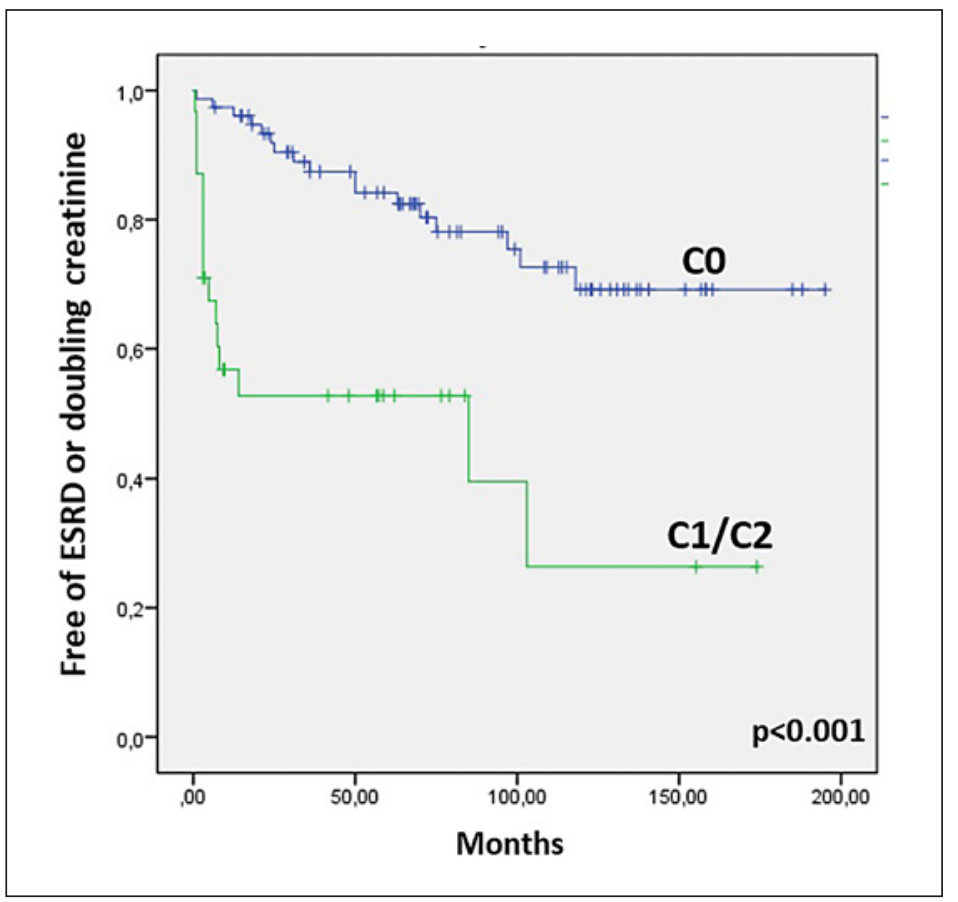

\begin{tabular}{llll}
\hline Variable & OR & $95 \%$ CI & $p$ value \\
\hline Male sex & 2.95 & $1.29-6.5$ & 0.01 \\
$\begin{array}{l}\text { Hypertension } \\
\text { Haematuria }\end{array}$ & 4.10 & $1.43-10.51$ & 0.005 \\
Oxford classification & 0.61 & $0.26-1.44$ & 0.27 \\
$\quad$ M1 & 1.44 & $0.55-4.04$ & 0.62 \\
E1 & 2.12 & $0.95-4.86$ & 0.08 \\
S1 & 5 & $1.46-16.59$ & 0.008 \\
T1/T2 & 4.8 & $1.91-11.9$ & 0.0007 \\
C1/C2 & 3.2 & $1.32-7.2$ & 0.01 \\
\hline
\end{tabular}

C1/C2, pronounced/severe crescent formation; CI, confidence interval; E1, pronounced endocapillary hypercellularity; ESRD, endstage renal disease; IgAN, immunoglobulin A nephropathy; M1, pronounced mesangial hypercellularity; OR, odds ratio; S1, pronounced segmental glomerulosclerosis; T1/T2, pronounced/severe tubular atrophy or interstitial fibrosis.

Table 4 shows the distribution of the composite outcome (ESRD or doubling of serum creatinine). Comparing the patients in whom the composite outcome was and was not observed, we found that those in the former group were younger ( 29 vs. 34 years, $p=0.003$ ). In addition, the proportion of men was higher in the composite outcome group (62.5 vs. $37.7 \%, p=0.01$ ), as was that of hypertensive patients (85.7 vs. $55.0 \%, p=0.0005)$. At admission, the mean serum creatinine concentration was also higher in the composite outcome group ( 2.7 vs. $1.2 \mathrm{mg} / \mathrm{dL}, p<0.0001$ ), whereas the mean CKD-EPI-determined eGFR was lower (36.6 vs. $66.8 \mathrm{~mL} / \mathrm{min} / 1.73 \mathrm{~m}^{2}$ ), as was the mean serum C3 level (110.7 vs. 125.5 $\mathrm{mg} / \mathrm{dL}, p=0.02$ ). At renal biopsy, the proportion of patients with an Oxford classification of $\mathrm{S} 1$ was higher in the composite outcome group (100.0 vs. $66.2 \%, p=0.0002)$, as was that of 


\section{Kidney \\ Blood Pressure \\ Research}

Table 6. Cox regression analysis for the composite outcome (ESRD or doubling of serum creatinine), adjusted for sex, proteinuria, eGFR by the Chronic Kidney Disease Epidemiology Collaboration, hypertension, haematuria, and Oxford classification

\begin{tabular}{l|l}
\hline Kidney Blood Press Res 2020;45:431-441 \\
\hline DOI: 10.1159/000507251 & $\begin{array}{l}\text { @ 2020 The Author(s). Published by S. Karger AG, Basel } \\
\text { www.karger.com/kbr }\end{array}$ \\
\hline
\end{tabular}

\begin{tabular}{llll}
\hline Variable & HR & $95 \% \mathrm{CI}$ & $p$ value \\
\hline $\begin{array}{llll}\text { Oxford classification } \\
\mathrm{S} 1\end{array}$ & 1.6 & $1.14-4.97$ & 0.04 \\
$\mathrm{~T} 1 / \mathrm{T} 2$ & 3.04 & $1.13-8.2$ & 0.027 \\
$\mathrm{C} 1 / \mathrm{C} 2$ & 2.43 & $1.08-5.45$ & 0.031 \\
\hline
\end{tabular}

$\mathrm{C} 1 / \mathrm{C} 2$, pronounced/severe crescent formation; CI, confidence interval; eGFR, estimated glomerular filtration rate; ESRD, end-stage renal disease; HR, hazard ratio; $\mathrm{S} 1$, pronounced segmental glomerulosclerosis; T1/T2, pronounced/severe tubular atrophy or interstitial fibrosis.

those with a classification of T1/T2 (78.1 vs. $25.9 \%, p<0.0001)$, that of those with a classification of $\mathrm{C} 1 / \mathrm{C} 2$ ( $46.8 \mathrm{vs} .20 .8 \%, p=0.006$ ), and that of those requiring treatment with immunosuppressants other than corticosteroids (33.3 vs. $13.7 \%, p=0.01$ ).

Survival free of the composite outcome was shorter among the patients in the C1/C2 group (Fig. 1), in which there was a 3.2-fold higher risk of that outcome $(95 \%$ confidence interval [CI] 1.32-7.2, $p=0.01$ ), as shown in Table 5. Other factors found to increase the risk of the composite outcome were being male, for which the odds ratio (OR) was 2.95 (95\% CI $1.29-6.5, p=0.01$ ), and being hypertensive (OR 4.1, 95\% CI 1.43-10.51, $p=0.005$ ) as well as having an Oxford classification of S1 (OR 5.0, 95\% CI 1.46-16.59, $p=0.008$ ) or T1/T2 (OR 4.8, $95 \% \mathrm{CI} 1.91-11.9, p=0.0007$ ). In the Cox regression analysis (Table 6), the independent risk factors for the composite outcome had Oxford classifications of C1/C2 (hazard ratio [HR] 2.43, 95\% CI 1.08-5.45, $p=0.031$ ), S1 (HR 1.6, 95\% CI 1.14-4.97, $p=0.04$ ), or T1/T2 (HR 3.04, $95 \%$ CI $1.13-8.2, p=0.027$ ).

\section{Discussion and Conclusion}

Although IgAN continues to be the most common primary glomerulopathy, its pathogenesis has begun to be understood only recently. Due to the high rate of progression from IgAN to ESRD, it is important to identify the clinical, biochemical, and histological factors that are related to a poorer prognosis in patients with IgAN. The clinical and biochemical factors that are associated with a poorer prognosis include uncontrolled hypertension, renal failure, severe proteinuria, smoking, hyperuricaemia, obesity, advanced age, and prolonged duration of the initial symptoms $[1,4,16]$.

The original version of the Oxford classification sought to determine which histopathological findings had an influence on the prognosis of IgAN $[9,10]$. In that version, the histological variables mesangial hypercellularity, segmental sclerosis, and tubular atrophy/interstitial fibrosis, independently of clinical factors, were associated with a poorer prognosis in IgAN, whereas endocapillary hypercellularity was associated with a decline in function only in patients treated with immunosuppressants. Vascular lesions and crescents were not included as potential prognostic factors, perhaps because patients with an eGFR $<30 \mathrm{ml} / \mathrm{min} / 1.73 \mathrm{~m}^{2}$ at the time of biopsy were excluded from the original Oxford sample and the development of ESRD within the 12 months thereafter was not evaluated.

Shortly after the publication of the Oxford classification in 2009, Katafuchi et al. [11] evaluated the impact of crescents on prognosis in a study of 702 patients with IgAN. The authors evaluated the development of ESRD within the first 12 months after biopsy. Although 
their sample included patients with an eGFR $<30 \mathrm{~mL} / \mathrm{min} / 1.73 \mathrm{~m}^{2}$ at the time of biopsy, such patients accounted for only $4 \%$ of the cases evaluated. The impact of crescents on the prognosis of IgAN has been demonstrated in univariate and multivariate models correlating the clinical and histological aspects.

Haas et al. [12] compiled data for a collective total of 3,096 adults and children evaluated in the Oxford study, in the validation IgA study, in a study conducted in Japan, and in a study conducted in China. Their sample included patients with an eGFR $<30 \mathrm{~mL} / \mathrm{min} / 1.73 \mathrm{~m}^{2}$ and a rapid course of progression to renal failure. The authors concluded that crescents in at least one-sixth of the glomeruli increased the risk of a composite outcome (progression to ESRD or a 50\% drop in eGFR). In addition, having cellular crescents in at least one-fourth of the glomeruli was significantly associated with the composite outcome, regardless of the use of immunosuppression.

In the present study, we chose to group the patients who had an Oxford classification of C1 together with those who had a classification of C2 (C1/C2 group) because only a small proportion of patients had a classification of $\mathrm{C} 2$, which would have precluded an appropriate statistical evaluation. In a study of 538 patients with IgAN in China, Zhang et al. [13] showed that the proportion of crescents in a biopsy specimen also affects the prognosis. The authors divided the patients into four groups by the proportion of glomeruli containing crescents: $<5 \%, 5-9 \%, 10-24 \%$, and $\geq 25 \%$. They observed no difference in prognosis between the patients with crescents in $<5 \%$ of the glomeruli and those without crescents. However, in the three remaining groups, the prognosis worsened in parallel with increases in the proportion of crescents.

The analysis of studies such as those cited above culminated in the 2016 update of the Oxford classification, in which crescents were also recognised as histological markers of poor prognosis. Such findings corroborate those of other studies regarding the role of crescents in the prognosis of IgAN, in adult $[17,18]$ and paediatric patients [19-21], as well as in cases of IgAN recurrence after kidney transplantation $[22,23]$.

The specific pathogenesis of crescent formation in IgAN has yet to be elucidated. In a study conducted in Japan, Sasaki et al. [24] evaluated the involvement of the tumour necrosis factor-like weak inducer of apoptosis (TWEAK) and its receptor, fibroblast growth factorinducible 14 (Fn14), in the formation of crescents in patients with IgAN. The TWEAK/Fn14 system has a proven proinflammatory effect on mesangial cells and podocytes through activation of nuclear factor-kappa $B$ and the consequent induction of expression of inflammatory cytokines such as interleukin 6 and chemokine CCL2. That also results in increased podocyte motility and mesangial proliferation. Sasaki et al. [24] evaluated clinical, histological, and cell culture findings, observing that the activation of the TWEAK/Fn14 system correlated with the formation of crescents, the proportion of crescents being directly proportional to the urinary level of soluble TWEAK. The authors also found that urinary TWEAK levels correlated with proteinuria in patients with IgAN.

Although there have been some studies evaluating the clinical, biochemical, and histological characteristics of patients with IgAN in Latin America [25-27], few such studies have followed those patients over time or evaluated the impact of crescents. In a previous study [28], we also evaluated the impact of crescents on the prognosis of patients with IgAN. In that study, we evaluated 144 patients with IgAN, 26 of whom had crescents. We found that the patients with crescents had a worse initial clinical presentation and a less favourable evolution, as well as showing a higher incidence of ESRD over a mean follow-up period of approximately 26 months.

Our study has as limitation, namely the fact that it is a retrospective and single-centred study; however, the uniqueness of a service guarantees better uniformity in data collection, histological analysis, treatment, and follow-up. 
The results of the present study underscore the importance of crescents in the prognosis of patients with IgAN. Our findings corroborate those of previous studies of such patients and add to the growing body of evidence regarding IgAN in Latin America.

\section{Statement of Ethics}

Ethics approval was not required for this study since it is a retrospective non-interventional study.

\section{Disclosure Statement}

The authors have no conflicts of interest to declare.

\section{Funding Sources}

University of São Paulo School of Medicine.

\section{Author Contributions}

P.D.M.M. Neves performed medical record research, analysed data, and wrote the manuscript. R.B.B. Pinheiro, L.A. Testagrossa, L.B. Cavalcante, and D.M.A.C. Malheiros performed histological analysis at its various levels and revised the final version of the manuscript. C.B. Dias and L. Yu performed patient follow-up and revised the final version of the manuscript. L.B. Jorge and V. Woronik performed patient follow-up, analysed data, wrote the manuscript, and revised the final version of the manuscript.

\section{References}

1 Rodrigues JC, Haas M, Reich HN. IgA Nephropathy. Clin J Am Soc Nephrol. 2017 Apr;12(4):677-86.

2 Trimarchi H, Barratt J, Monteiro RC, Feehally J. IgA nephropathy: "State of the art": a report from the 15th International Symposium on IgA Nephropathy celebrating the 50th anniversary of its first description. Kidney Int. 2019 Apr; 95(4):750-6.

3 D'Amico G. Natural history of idiopathic IgA nephropathy and factors predictive of disease outcome. Semin Nephrol. 2004 May;24(3):179-96.

4 Wyatt RJ, Julian BA. IgA nephropathy. N Engl J Med. 2013 Jun;368(25):2402-14.

5 Li LS, Liu ZH. Epidemiologic data of renal diseases from a single unit in China: analysis based on 13,519 renal biopsies. Kidney Int. 2004 Sep;66(3):920-3.

6 Hall CL, Bradley R, Kerr A, Attoti R, Peat D. Clinical value of renal biopsy in patients with asymptomatic microscopic hematuria with and without low-grade proteinuria. Clin Nephrol. 2004 Oct;62(4):267-72.

7 Donadio JV, Grande JP. IgA nephropathy. N Engl J Med. 2002 Sep;347(10):738-48.

8 Maixnerova D, Reily C, Bian Q, Neprasova M, Novak J, Tesar V. Markers for the progression of IgA nephropathy. J Nephrol. 2016 Aug;29(4):535-41.

9 Cattran DC, Coppo R, Cook HT, Feehally J, Roberts IS, Troyanov S, et al.; Working Group of the International IgA Nephropathy Network and the Renal Pathology Society. The Oxford classification of IgA nephropathy: rationale, clinicopathological correlations, and classification. Kidney Int. 2009 Sep;76(5):534-45.

10 Roberts IS, Cook HT, Troyanov S, Alpers CE, Amore A, Barratt J, et al.; Working Group of the International IgA Nephropathy Network and the Renal Pathology Society. The Oxford classification of IgA nephropathy: pathology definitions, correlations, and reproducibility. Kidney Int. 2009 Sep;76(5):546-56.

11 Katafuchi R, Ninomiya T, Nagata M, Mitsuiki K, Hirakata H. Validation study of oxford classification of IgA nephropathy: the significance of extracapillary proliferation. Clin J Am Soc Nephrol. 2011 Dec;6(12):2806-13. 
12 Haas M, Verhave JC, Liu ZH, Alpers CE, Barratt J, Becker JU, et al. A Multicenter Study of the Predictive Value of Crescents in IgA Nephropathy. J Am Soc Nephrol. 2017 Feb;28(2):691-701.

13 Zhang W, Zhou Q, Hong L, Chen W, Yang S, Yang Q, et al. Clinical outcomes of IgA nephropathy patients with different proportions of crescents. Medicine (Baltimore). 2017 Mar;96(11):e6190.

14 Trimarchi H, Barratt J, Cattran DC, Cook HT, Coppo R, Haas M, et al.; IgAN Classification Working Group of the International IgA Nephropathy Network and the Renal Pathology Society; Conference Participants. Oxford Classification of IgA nephropathy 2016: an update from the IgA Nephropathy Classification Working Group. Kidney Int. 2017 May;91(5):1014-21.

15 Levey AS, Stevens LA, Schmid CH, Zhang YL, Castro AF 3rd, Feldman HI, et al.; CKD-EPI (Chronic Kidney Disease Epidemiology Collaboration). A new equation to estimate glomerular filtration rate. Ann Intern Med. 2009 May;150(9):604-12.

16 Barbour S, Reich H. An update on predicting renal progression in IgA nephropathy. Curr Opin Nephrol Hypertens. 2018 May;27(3):214-20.

17 Shao X, Li B, Cao L, Liang L, Yang J, Wang Y, et al. Evaluation of crescent formation as a predictive marker in immunoglobulin A nephropathy: a systematic review and meta-analysis. Oncotarget. 2017 Jul;8(28):4643648.

18 Peng W, Tang Y, Tan L, Qin W. Crescents and Global Glomerulosclerosis in Chinese IgA Nephropathy Patients: A Five-Year Follow-Up. Kidney Blood Press Res. 2019;44(1):103-12.

19 Edström Halling S, Söderberg MP, Berg UB. Predictors of outcome in paediatric IgA nephropathy with regard to clinical and histopathological variables (Oxford classification). Nephrol Dial Transplant. 2012 Feb;27(2): 715-22.

20 Rianthavorn P, Chacranon M. Long-term renal outcome in pediatric glomerulonephritis associated with crescent formation. Clin Exp Nephrol. 2018 Jun;22(3):661-7.

21 Wang D, Wang F, Ding J, Xiao H, Zhong X, Liu X. Retrospective study of primary IgA nephropathy with crescent formation and/or rapidly progressive glomerulonephritis in children. Zhonghua Er Ke Za Zhi. 2015 Sep;53(9): 670-5. Chinese.

22 Guo JQ, Song BL, Wu ZX, Wu WZ, Luo LT, Chen XW, et al. Prognostic factors for renal allograft survival in patients with immunoglobulin A nephropathy: a case control study. Mol Med Rep. 2014 Apr;9(4):1179-84.

23 Park S, Baek CH, Cho H, Yu MY, Kim YC, Go H, et al. Glomerular crescents are associated with worse graft outcome in allograft IgA nephropathy. Am J Transplant. 2019 Jan;19(1):145-55.

24 Sasaki Y, Shimizu Y, Suzuki Y, Horikoshi S, Tomino Y. TWEAK/Fn14 system and crescent formation in IgA nephropathy. BMC Nephrol. 2015 Mar;16:27.

25 Neves PD, Machado JR, Silva MV, Abate DT, Rodrigues DB, Faleiros AC, et al. IgA nephropathy: histological analysis and clinicomorphological correlation in patients from Minas Gerais State. J Bras Nefrol. 2012 Jun; 34(2):101-8.

26 Soares MF, Caldas ML, Dos-Santos WL, Sementilli A, Furtado P, Araújo S, et al. IgA nephropathy in Brazil: apropos of 600 cases. Springerplus. 2015 Sep;4:547.

27 Souza BN, Tavares MB, Soares MF, Santos WL. IgA Nephropathy in Salvador, Brazil. Clinical and laboratory presentation at diagnosis. J Bras Nefrol. 2018 Jul-Sep;40(3):242-7.

28 Bitencourt-Dias C, Bahiense-Oliveira M, Saldanha LB, Barros RT, Woronik V. Comparative study of IgA nephropathy with and without crescents. Braz J Med Biol Res. 2004 Sep;37(9):1373-7. 